\title{
MULTIWAVELENGTH ENERGY DISTRIBUTIONS OF ULTRALUMINOUS IRAS GALAXIES
}

\author{
D.RIGOPOULOU and A.LAWRENCE \\ Physics Dept., QMW, Mile End Rd., London E1 4NS, U.K.
}

\begin{abstract}
Ultraluminous IRAS Galaxies (ULG's) have luminosities comparable to quasars while their space density is much higher than that of active galaxies. Much debate has centered around the origin of the energy source for these objects, whether this is a burst of star formation or a hidden quasar. The sample studied here is the Sanders et al.(1988) sample, 10 objects with $L_{F I R} \geq 10^{12} L_{\odot}$. We discuss our new observations at $\mathrm{X}$-ray and submm wavelengths together with other published data for some of the objects [1]. Some useful ideas can be gained from comparisons of the shape of the spectral energy distributions (SED's) of the ultraluminous objects with other "archetype" objects such as typical starbursts i.e. M82 or type 2 AGN i.e. NGC1068.
\end{abstract}

05189-2524:

ROSAT data for this galaxy revealed a strong source with a count rate of $0.039 \mathrm{cnts} / \mathrm{sec}$. We fitted the spectrum with a simple model, the absorbed power law, with an energy index $\alpha=1.28 \pm 0.37$. The SED of 05189-2524 is compared with that of NGC 1068. Emission from the starburst disk dominates at longer wavelengths while at 20 microns and shortward nuclear emission starts to dominate spectrum. Judging from the shape of the SED of 05189-2524 we may argue that a similar situation holds for this galaxy although the relative strength of the two components is not the same as in NGC 1068. This might be a more evolved system where the starburst episode has started to fade away and the Seyfert nucleus starts to emerge through the dust. We must also stress the fact that the emission lines of 05189 look like those of Seyfert 2 [2] and we find a relatively strong $\mathrm{X}$-ray emission.

14348-1447:

Our ROSAT data give evidence for a weak source. We achieved a $3 \sigma$ detection at the radio position. The multiwavelength energy distribution of 14348-1447 is compared with that of ARP220. The shapes of the two SED's are different especially in the optical-UV area. The rise in the SED of ARP 220 at the optical-UV region which is due to the hot newly formed stars is not observed in the SED of 14348. Different degrees of obscuration or a different energy source might be the cause of this discrepancy.

\section{$15250+3609$ :}

From our ROSAT data there is evidence for a $2.5 \sigma$ source at the radio position with a low count rate of $2.7 \times 10^{-3}$ counts/sec. The object is compared with the starburst galaxy M82. The overall shape is pretty similar but the ratio $S_{60 \mu \mathrm{m}} / S_{1 \mathrm{KeV}}$ is different. The above ratio is equal to $3.12 \times 10^{8}$ for $\mathrm{M82}$ (consistent with the values found by [3]) compared to $2.48 \times 10^{9}$ for $15250+3609$. If a starburst episode is responsible for the amounts of energy released at IR wavelengths then these bursts must occur at the inner parts of the galaxy and are hidden from our sight of view by massive molecular clouds and gas, the latter absorbing all the soft $x$-rays.

\section{References}

1. Rigopoulou, D., and Lawrence, A. 1993, in preparation.

2. Sanders, D.B., Soifer, B.T., Elias, J.H., Madore, B.F., Matthews, K., Neugebauer, G., and Scoville N.Z. 1988, Ap.J., 325,74

3. Weedman, D.W. 1987 in Star Formation in Galaxies, ed. C.J.Persson (Washington, DC:US Government Printing Office)

T. J.-L. Courvoisier and A. Blecha: Multi-Wavelength Continuum Emission of AGN, 332.

(C) 1994 IAU. Printed in the Netherlands. 